\title{
Plant-Endophyte Interaction and Its Application to Abiotic Stress Management of Crop Plants
}

\author{
Prajjal Dey $^{1}$, Diptanu Datta $^{2 *}$, Debanjana Saha $^{3}$, Selukash Parida $^{1}$ and Darshan Panda ${ }^{1}$ \\ ${ }^{1}$ Department of Crop Physiology and Biochemistry, National Rice Research Institute, \\ Cuttack, India \\ ${ }^{2}$ Department of Plant Pathology, Odisha University of Agriculture and Technology, \\ Bhubaneswar, India \\ ${ }^{3}$ Department of Agricultural Biotechnology, Odisha University of Agriculture and \\ Technology, Bhubaneswar, India \\ *Corresponding author
}

\section{A B S T R A C T}

Endophytes are microorganisms mostly bacteria and fungi that lives partially or completely its life cycle inside living plant tissue without causing adverse negative symptoms. Endophytes play a major role in structuring shaping processes of plant such as

\begin{tabular}{|l|}
\hline Ke y w or d s \\
Endophytes, \\
Mutualistic/Symbio \\
tic, Abiotic stress, \\
Crop plants \\
\hline Article Info \\
\hline $\begin{array}{l}\text { Accepted: } \\
\text { 20 June 2019 } \\
\text { Available Online: } \\
\text { 10 July 2019 }\end{array}$ \\
\hline \hline
\end{tabular}
colonization, competition, coexistence and soil nutrient dynamics. Some endophytes promote growth of their host plants by enhancing the host's ability to take up nitrogen and phosphorus. Recently endophytic fungi have gained importance in agriculture because of their ability to confer resistance to different abiotic stress conditions like temperature, cold, light intensity, drought, salinity etc., and biotic stress conditions like pathogen attack and insect herbivory by aiding several plant growth processes. Trichoderma can promote plant growth and also suppress the pathogen by producing numerous metabolites. They also induce systemic resistance against disease, they also bring about morphological adaptation which help in overcoming drought stress. Endophytic fungi Fusarium culmorum (FcRed1) found to have symbiotic relationship with rice seedlings under salinity and drought which in turn helps in increasing plant height, root length, biomass, higher osmolyte accumulation than the nonsymbiotic rice seedlings. Different fungal endophyte were known to confer thermo tolerance and produce different heat shock protein while living inside the plant and thereby helping the plants to sustain under conditions of both high and low temperature. This review will briefly summarize about plant-endophyte interaction and also its application in mitigating abioic stress in crop plants.

\section{Introduction}

Endophytes ("endo" = within, "phyte" = plant), are microorganisms (fungal and bacterial) that lives part of its life or complete its life cycle inside living plant tissue without causing overt negative symptoms. The distribution of endophytes is ubiquitous and almost without exception the endophytes have been reported from all tissues, including leaves, stems, roots, flowers and fruits. Endophytes ("Entophytae") were first 
described by the German botanist Heinrich Friedrich Link in 1809. Every plant examined to date harbours at least one species of endophytic fungus and many plants, especially woody plants may contain literally hundreds or thousands of species (Petrini et al., 1992; Gaylord et al., 1996; Faeth and Hammon. 1997 and Arnold et al., 2000).

Dutch microbiologist Martinus Willem Beijerinck (1888) isolated root nodule bacteria in pure culture from nodules of Leguminosae plants capable of fixing atmospheric nitrogen which were later classified as Rhizobium leguminosarum. Phenomenon of symbiotic nitrogen fixation by rhizobia and mineral $\mathrm{N}$ independence in legume plants were reported by Hermann Hellriegel and Hermann Wilfarth. Albert Bernhard Frank (1888) reported another important mutualistic symbiosis, i.e., the living together of unlike organisms, between roots of trees and underground fungi. $\mathrm{He}$ coined the term "mycorrhiza" to describe the interaction, which literally means "fungus roots."

Endophytic fungi are classified into class I endophytes, referred as clavicipitaceous endophytes which are restricted to grass species (Bischoff et al., 2005); they live their entire life cycle within the aerial portion of the host grass, forming non-pathogenic, systemic and usually intercellular associations (Arora et al., 1991). Class I endophytes, are reported to increase plant biomass, confer drought tolerance (Clay et al., 1988). These are vertically transferred through seeds from one generation to other generation. Class II endophytes are called as non-clavicipitaceous. They are mutualistic/symbiotic and confer benefits to hosts during abiotic stress and obtain nutrition for growth and reproduction to host tissues (Rodriguez et al., 2009). A very unique and fascinating trait of these endophytes is that they confer habitat-specific stress tolerance to plants through a process defined as "Habitat Adapted Symbiosis" (Rodriguez et al., 2008). Remarkably, Class II endophytes, are capable of colonizing and conferring habitat-specific stress tolerance to monocot and eudicot plants which may suggest that the symbiotic communication responsible for stress tolerance may predate the divergence of these lineages (Rodriguez et al., 2008).

Endophytic fungi play a major role in structuring plant communities and in shaping processes such as colonization, competition, coexistence and soil nutrient dynamics (Schulz et al., 2002). Some endophytes promote growth of their host plants by enhancing the host's ability to take up nitrogen and phosphorus (Malinowski and Belesky, 1999).

Endophytic fungi have gained importance in the area of agriculture because of their ability to confer resistance to different abiotic stress conditions like temperature, cold, light intensity, drought, salinity etc., and biotic stress conditions like pathogen attack and insect herbivory by aiding several plant growth processes (Bae et al., 2009 and Arnold et al., 2003).

There are reports that both class I and class II endophytes have important role in abiotic stress tolerance (Rodriguez et al., 2008). In recent years there is a growing interest to understand the role of endophytic fungi in imparting tolerance to host plants. Several questions have been addressed: Are endophytes specific to their host plants and their habitat? Can these endophytes be successfully used to mitigate abiotic stress in crop plants? With this background, we can discuss the role and mechanism of endophytes in abiotic stress tolerance and their probable formulations for practical applications in the field. 


\section{Abiotic Stress}

Abiotic stress is the major threat to agriculture, leading to decreased productivity by more than $50 \%$ in major crop plants word wide (Bray et al., 2000). Abiotic stress, affects plant growth and productivity by morphological, physiological, biochemical and molecular changes in plant (Wang et al., 2001). Abiotic stress such as drought, salinity, high temperatures and oxidative stress are often interconnected leading to cellular damage and cell death ultimately (Wang et al., 2003). Since a decade or so, Perusal of literature has shown that fungal endophytes confer stress tolerance to host species and play a significant role in the survival of plants in abiotic stress environments (Rodriguez et $a l ., 2009)$ and this can be an alternate strategy to overcome the abiotic stress in plants.

\section{Drought}

Drought stress induces a range of physiological and biochemical responses in plants such as stomatal closure, repression of growth and photosynthesis, and activation of respiration. The root-colonizing endophyte Pirifomospora indica was shown to confer drought tolerance in Arabidopsis and Chinese cabbage by stimulating the expression of drought stress-related genes in leaves (Sun et al., 2010). Piriformospora indica, an endophytic fungus cocultivated with Arabidopsis plants showed earlier upregulation of mRNAs and proteins involved in drought stress from leaves of colonized than the uncolonized Arabidopsis seedlings. $P$. indica colonized roots of Chinese cabbage, promotes root and shoot growth, and promotes lateral root formation. When $P$. indica colonized plants were exposed to polyethylene glycol to mimic drought stress, the activities of peroxidases, catalases and superoxide dismutases in the leaves were up regulated within 24 hours. The fungus retarded the drought-induced decline in the photosynthetic efficiency and the degradation of chlorophylls and thylakoid proteins (Sun et al., 2010).

Trichoderma hamatum isolate DIS 219b, an endophyte isolated from Theobroma gileri was reported to promote growth and delay the onset of drought response in Theobroma cacao (Bae et al., 2009).

Malinowski et al., (1999b) showed that endophyte infection increased root hair length and decreased root diameter in tall fescue which have role in water and mineral acquisition. White et al., (1992) showed that endophyte infection lowered bulk modulus of tissue elasticity (suggesting less rigid cell walls) and increased turgid weight: dry weight (TW/DW) ratio in water stressed tall fescue. Symbiotic plants consumed significantly less water than non-symbiotic plants regardless of the colonizing endophyte. Panic grass, rice, tomato and dune grass plants all used significantly less fluid than non-symbiotic plants. This study indicated that endophytic fungi help plants to use the water efficiently under stress conditions (Malinowski and Belesky, 2000). Work on non-clavicipitaceous endophytes showed that endophytes either scavenge ROS, induces plants to more efficiently scavenge ROS or prevents ROS production when exposed to abiotic stress (Rodriguez et al., 2008).

\section{Salinity}

Salinity is a major abiotic stress that restricts yield on almost 40 million hectares of irrigated land, which is approximately onethird of the irrigated land on earth. It is estimated that about $50 \%$ of the arable land will be affected by salinity stress by the year 2050. Khan et al., (2011a; b) confirmed that GA producing endophytic fungal strains ( $P$. funiculosum and Aspergillus fumigatus) can 
ameliorate soybean plant growth under moderate and high salinity stress. Paecilomyces formosus LHL10 inoculation significantly improved plant growth and alleviated salinity induced stress (Khan et al., 2012).

Salinity severely affects plant growth and metabolism resulting in reduced biomass production. Plants have the capability to cope with these stresses through many signal transduction pathways adjusting their metabolism (Kasuga et al., 1999; Hasegawa et al., 2000 and Xiong et al., 2002). Sodium ion toxicity trigger the formation of reactive oxygen species (ROS) such as superoxide $\left(\mathrm{O}_{2}^{-}\right)$, hydrogen peroxide $\left(\mathrm{H}_{2} \mathrm{O}_{2}\right)$, and hydroxyl radical $(\bullet \mathrm{OH})$ which ultimately affect (i) mitochondria and chloroplasts, (ii) water use efficiency, (iii) photosynthesis, and (iv) nutrients uptake whilst disrupting cellular structures (Munns et al., 2008). To avoid oxidative damage, plants adapt the mechanism of de novo synthesis of osmolytes like proline, sorbitol, mannitol etc. Several studies have indicated that symbiotic association of endophytic fungi can ameliorate plant growth and its physiological status. Fungal endophyte (Fusarium culmorum) was isolated from tissues and seed coats of Leymusmollis and was shown to contribute to salt tolerance of the plant (Rodriguez et al., 2008).

Redman et al., (2011) isolated the endophytic fungi Fusarium culmorum (FcRed1) from coastal beach and evaluated its role in cultivated rice varieties var. M-206 (subspecies Japonica) and Dongjin (subspecies Indica). The results showed that the symbiotic rice seedlings under stress conditions such as salinity and drought performed better with respect to plant height, root length, biomass, higher osmolyte accumulation than the nonsymbiotic rice seedlings indicating that Fusariumculmorum has a role in mitigating salt and drought stress.

\section{Temperature}

A temperature is a comparative objective measure of hot and cold. It is measured, typically by a thermometer. Temperature is one among the abiotic stress that severely affects plant growth and metabolism resulting in reduced biomass production and plant death. Plants have developed few mechanisms to cope with these stresses through many signal transduction pathways adjusting their metabolism (Kasuga et al., 1999; Hasegawa et al., 2000 and Xiong et al., 2002). Temperature stress could be either low temperature stress or High temperature stress.

\section{Low temperature}

Low temperatures can result in poor growth. Photosynthesis is slowed down at low temperatures. Since photosynthesis is slowed, growth is slowed, and this results in lower yield. Low temperature stress has also been shown to increase the $\mathrm{H}_{2} \mathrm{O}_{2}$ accumulation in cells. Cold stress tolerance was conferred to germinated seeds under laboratory conditions by TempSym1 (Curvularia protuberata) isolated from Dichanthelium lanuginosum thriving in geothermal soils (Redman et al., 2011).

\section{High temperature}

Crop plants are highly sensitive to ambient temperature, with a $1^{\circ} \mathrm{C}$ difference in temperature sufficient to affect development and yield. Monocot crop plants are particularly vulnerable to higher temperatures during the reproductive and grain-filling phases. The critical temperature above which plants get killed is called thermal 'death point'. The temperature above $50^{\circ} \mathrm{C}$ kills many annual crops. High temperature 
adversely affects mineral nutrition acquisition or uptake, shoot growth and pollen development and disturbs the photosynthesis and respiration processes resulting in low yield. As temperatures rise, production of heat shock proteins increases. Metabolic imbalances associated with temperature extremes result in the build-up of reactive oxygen species. Cell membranes are also affected by changes in temperature and can cause the membrane to lose its fluid properties becomes leaky in hot conditions. This can affect the movement of compounds across the membrane.

Fungal endophyte (Curvularia protuberata) conferred thermotolerance (over a 12-month field trial and under laboratory conditions) to the monocot Dichanthelium lanuginosum (panic grass), which thrives in geothermal soils of Yellowstone National Park, Wyoming (YNP) (Redman et al., 2002; Rodriguez et al., 2008). Curvularia protuberate (Cp4666D) conferred heat tolerance and hence plant survival to both tomato and panic grass plants (Rodriguez et al., 2008).

Fungal endophytes can also control plant responses to stress through the production of specific molecules. Paraphaeosphaeri aquadriseptata produces a heat shock protein 90 (HSP90) inhibitor, monocillin I, which enhances heat stress tolerance in Arabidopsis (Mclellanet al., 2007).

Suryanarayanan et al., (2011), showed that mesophilic fungal spores isolated from leaf litters of semi-arid regions of Western Ghats showed thermo-tolerance when exposed to dry season fire. This extreme thermotolerance of the fungal spores even at $100^{\circ} \mathrm{Cto}$ $115{ }^{0}$ Cshows that this physiological trait is more widespread than recognized.

Symbiotically conferred stress tolerance by endophytic fungus to the plants involves different mechanisms. Upregulating host stress response systems soon after exposure to stress, (Schulz et al., 1999 and Redman et al., 1999), or biosynthesis of anti-stress biochemical by endophytes (Schulz et al., 2002 and Miller et al., 2002) are the two major categories of the mechanism developed by endophytic fungus to protect plants from abiotic and biotic stress.

\section{Mechanism of tolerance by endophytes}

Past efforts to improve plant tolerance to abiotic stress through breeding and genetic engineering have had limited success owing to the genetic complexity of stress responses. Progress is now anticipated through plant endophyte interaction by which endophytes impart tolerance to plants in a habitat-specific manner (Rodriguez et al., 2008). This feature, referred to as "habitat-adapted symbiosis", has now been widely recognized in laboratory. The endophytes from these habitats confer habitat-specific stress tolerance to plants. This habitat-specific phenomenon provides an inter-genomic epigenetic mechanism for plant adaptation and survival in high-stress habitats (Redman et al., 2002a; Rodriguez et al., 2008). In order to design habitat adapted symbiosis, we must first develop an understanding of the mechanism underlying by which endophytes impart tolerance to host plant. Examples of hypotheses for the abiotic stress tolerance mechanism include:

Oxidative stress protection by increased production of antioxidants produced either by the microbes or by hosts in response to microbes;

Ethylene reduction by production of ACC deaminase;

Ammonia or ammonium detoxification and consequent oxidative stress avoidance. 
Oxidative stress protection by increased production of antioxidants produced either by the microbes or by hosts in response to microbes

Oxidative stress protection via antioxidants: This hypothesis came about because it was recognized that many endophytes cause an increased expression of antioxidant enzymes and antioxidant phenolic compounds in plants. Increased production of antioxidants is important because the result of many abiotic stress events is an increase in reactive oxygen in plant tissues (White and Torres, 2009). Endophyte-induced increases in antioxidants would counteract stress-induced reactive oxygen. Waller et al., (2005) demonstrated that in the infection of barley roots with the fungal endophyte Priformospora indica the host shows an increase in anti-oxidative capacity due to activation of the host's glutathione-ascorbate cycle. White and Torres (2009) suggested that oxidative stress avoidance through production of antioxidative compounds could account for Epichloë-endophyte enhanced stress tolerance in host grasses.

The production of antioxidant substances by many endophytic microbes (Schulz et al., 2002; Huang et al., 2007) seems to provide further support for this hypothesized mechanism. Hamilton et al., (2012) reviewed literature on diverse types of fungal endophytes in diverse host plants and found support for the hypothesis that endophyte mediation of reactive oxygen may play a key role in endophyte induced changes in host plant oxidative stress susceptibility. Hamilton and Bauerle (2012) further showed that antioxidant activity in endophyte containing host plants was elevated under abiotic stress compared to endophyte-free hosts. These authors hypothesized that abiotic stress protection was largely a function of oxidative stress protection.

\section{Ethylene reduction by production of $\mathrm{ACC}$ deaminase}

Enhanced stress tolerance through ethylene regulation from research on the effects of plant growth promoting bacteria (PGPB) on plants an elegant mechanism for enhanced stress tolerance was proposed to explain increases in abiotic stress tolerance (Saraf et al., 2014). This mechanism is based on the production of excess ethylene when plants are under stress. Normal levels of ethylene are important for proper growth regulation in plants; stress-generated ethylene in high concentrations results in plant growth inhibition, although the precise mechanism is not clear (Saravanakumar and Samiyappan, 2007). Some bacterial endophytes that enhance stress tolerance produce an enzyme ACC deaminase that degrades the precursor of ethylene, 1-aminocyclopropane-1carboxylic acid (ACC). Degradation of ACC releases ammonia and $\alpha$-ketobutyrate and prevents formation of ethylene. ACC deaminase appears to be among the metabolites produced by some nonpathogenic bacterial and fungal symbionts of plants. Microbe-produced ACC deaminase cleaves ACC leaving the microbe with important nutrients to support growth.

It has been proposed that growth stimulation of plants bearing some bacteria may be the result of ACC deaminase activity where the bacteria remove ethylene that represses plant growth, permitting growth stimulation that result from auxin secretion by the microbes. However, experiments that involved comparing performance of plants transformed with genes to express ACC deaminase to nontransformed plants bearing symbiotic bacteria under stress conditions showed that nontransformed plants with bacteria outperformed transformed plants (Saraf 21 et al., 2014). This seems to indicate that the mechanism of microbe-enhanced stress 
tolerance and plant growth stimulation may involve more than ACC deaminase.

\section{Ammonia or ammonium detoxification and consequent oxidative stress avoidance}

This mechanism involves ammonia/ammonium scavenging by endophytic or 3 epiphytic microbes. Most fungi and bacteria that associate with plants have ammonia 4 transporters that they may use to absorb free ammonia from host tissues and cells. 5 Ammonia is one product that builds up in tissues during photorespiration when levels of 6 oxygen in plants increase and carbon dioxide levels fall. High levels of ammonia that are 7 generated during photorespiration in tissues results in generation of high levels of 8 reactive oxygen and oxidative stress (Kiraly et al., 2013). The high affinity of some 9 microbial endophytes for ammonia (White et al., 2015) makes them ideal 10 scavengers/detoxifiers of excess ammonia. Reduction in levels of ammonia in plant 11 tissues and cells would have the consequence of reducing oxidative stress in plants. One 12 interesting aspect of this hypothesized mechanism along with the previous ethylene 13 regulation mechanism is that both mechanisms actually relate to mechanisms whereby 14 the microbe extracts organic nitrogen from host cells. It is reasonable to expect to see a 15 flow of nutrients from plant to microbe and that excesses of any nutrient might be drained 16 off by the microbial symbionts, resulting in host cell detoxification

\section{Stress tolerance through endophyte- mediated osmotic adjustment}

One of the early ideas regarding endophyteenhanced tolerance to drought or salt stress was that it involved osmotic adjustment (Arachevaleta et al., 1989; Elmi and West, 1995). Osmotic adjustment is the capacity of plant cells to adjust their cytoplasmic osmotically active solutes in order to maintain turgor pressure in response to dehydration stress (DaCosta and Huang, 2006). In tall fescue infected by the endophytic fungus 101 Epichlö coenophiala osmotic adjustment was measured to occur in tiller meristems of 2 the grass during periods of drought, enabling the grass to resume growth more quickly 3 after the cessation of the period of drought (Elmi and West, 1995). The significance of 4 endophyte-mediated enhanced drought tolerance in tall fescue is that it enabled 5 cultivation of the grass in drought prone areas where the grass could not be cultivated 6 without the fungal endophyte. It has been proposed that secreted fungal endophyte 7 alkaloids such as lolines or secreted fungal sugar alcohols (e.g., mannitol or arabitol) may 8 be partially responsible for increased osmotic adjustment capacity in tall fescue grass 9 (Richardson et al., 1992). 10 Endophyte-enhanced salt stress tolerance may also relate to the osmotic 11 adjustment mechanism.

Recently, Gond et al., (2015) showed that a rhizobacterium 12 Pantoea agglomerans conferred tolerance to salt stress in tropical corn and demonstrated 13 up-regulation of several plant aquaporin genes. The aquaporins are proteins that function 14 in water flow through cell membranes (Peng et al., 2007). It is logical that aquaporins 15 and water movement between cells and the apoplast would be critical in adjusting 16 osmotic potentials in plant tissues (Peng et al., 2007). In this respect it is interesting to 17 note that $P$. agglomerans has been demonstrated to produce and secrete 1, 3-propanediol, 18 a compound that is osmotically active (Barbirato et al., 1996). Whether 1, 3propanediol 19 or other secreted metabolites could be osmoprotectants, inducing osmotic adjustment in 20 tropical corn has not been ascertained. 
Poly-mechanistic microbe-enhanced abiotic Stress tolerance in plant

The mechanisms for microbe-enhanced abiotic stress tolerance previously described could be considered competing hypotheses. On the other hand, they may not be mutually exclusive and multiple mechanisms could be in effect to induce abiotic stress tolerance. In this model for microbe-induced abiotic stress tolerance, multiple mechanisms could be in operation to differing degrees during stress events to ameliorate negative effects on plant hosts. Different mechanisms could be in operation depending on the microbes involved. For example, nitrifying bacteria that associate with roots may be particularly adept at capturing ammonia and converting it to nitrate. Nitrifiers could be working via the ammonia detoxification mechanism in plants that grow in aquatic habitats where ammonia may accumulate in high concentrations (Kiraly et al., 2013). Most microbes that associate with plants produce auxin. In many of these plant- associated microbes, ACC deaminase could be produced to degrade ACC as one avenue whereby microbes can extract nutrients from plants. Abiotic stress incited by soil heavy metals could be counteracted by antioxidants produced directly by microbes or by hosts in response to microbes. In situations where drought severely restricts plant growth osmotic adjustment facilitated by microbes might protect fragile meristems in which microbes grow so that recovery may occur rapidly once the drought ends. It seems likely that microbe-induced abiotic stress tolerance could be the result of more than one mechanism. Multiple mechanisms could explain the "habitat-adapted stress tolerance symbiosis phenomenon' described by Rodriguez et al., (2008) where microbes adapt host plants to particular stresses in the environment of the host. Using the example provided by Rodriguez et al., (2008), the mechanisms that adapt a plant to tolerate heat stress may be different from the combination of mechanisms that adapt a plant to tolerate salt stress

\section{References}

Arnold, A.E., Maynard, Z., Gilbert, G.S., Coley, P.D. and Kursar, T.A., 2000. Are tropical fungal endophytes hyperdiverse? Ecology letters. 3(4): pp.267-274.

Arnold, A.E., Mejía, L.C., Kyllo, D., Rojas, E.I., Maynard, Z., Robbins, N. and Herre, E.A., 2003. Fungal endophytes limit pathogen damage in a tropical tree. Proceedings of the National Academy of Sciences. 100(26): pp. $15649-15654$.

Arora, D.K., 1991. Handbook of Applied Mycology: Volume 1: Soil and Plants. CRC Press.

Bae, H., Sicher, R.C., Kim, M.S., Kim, S.H., Strem, M.D., Melnick, R.L. and Bailey, B.A., 2009. The beneficial endophyte Trichoderma hamatum isolate DIS 219b promotes growth and delays the onset of the drought response in Theobroma cacao. Journal of experimental botany. 60(11): pp.3279-3295.

Beijerinck, M.W., 1888. Cultur des Bacillus radicicola aus den Knöllchen. Bot Ztg, 46, pp.740-750.

Bischoff, J.F., 2005. In, DightonJ, White JF, Oudemans P, eds.

Bray, E.A., 2000. Response to abiotic stress. Biochemistry and molecular biology of plants. pp. 1158-1203.

Clay, K., 1988. Fungal endophytes of grasses: a defensive mutualism between plants and fungi. Ecology. 69(1): pp. 10-16.

Faeth, S.H. and Hammon, K.E., 1997. Fungal endophytes in oak trees: long- term patterns of abundance and associations with leafminers. Ecology. 78(3): pp.810-819. 
Frank, B., 1890. Ueber die Pilzymbiose der Leguminosen. P. Parey.

Gaylord, E.S., Preszler, R.W. and Boecklen, W.J., 1996. Interactions between host plants, endophytic fungi, and a phytophagous insect in an oak (Quercus grisea $\mathrm{X}$ Q. gambelii) hybrid zone. Oecologia. 105(3): pp. 336-342.

Hellriegel, H. and Wilfarth, H., 1888. Untersuchungen uber die Stickstoffnahrung der Gramineen und Leguminosen.

Malinowski, D.P. and Belesky, D.P., 2000. Adaptations of endophyte-infected coolseason grasses to environmental stresses: mechanisms of drought and mineral stress tolerance. Crop Science. 40(4): pp. 923-940.

Petrini, O., 1992. Fungal endophytes of bracken (Pteridium aquilinum), with some reflections on their use in biological control. Sydowia. 44: pp. 282-293.

Rodriguez, R.J., Henson, J., Van Volkenburgh, E., Hoy, M., Wright, L., Beckwith, F., Kim, Y.O. and Redman, R.S., 2008. Stress tolerance in plants via habitat-adapted symbiosis. The ISME journal. 2(4): p.404.

Rodriguez, R.J., White Jr, J.F., Arnold, A.E. and Redman, A.R.A., 2009. Fungal endophytes: diversity and functional roles. New phytologist, 182(2): pp. 314330.

Schulz, B., Boyle, C., Draeger, S., Römmert, A.K. and Krohn, K., 2002. Endophytic fungi: a source of novel biologically active secondary metabolites. Mycological research 106(9): pp. 9961004.

Sun, C., Johnson, J.M., Cai, D., Sherameti, I., Oelmüller, R. and Lou, B., 2010. Piriformospora indica confers drought tolerance in Chinese cabbage leaves by stimulating antioxidant enzymes, the expression of drought-related genes and the plastid-localized CAS protein. Journal of plant physiology. 167(12): pp. 1009-1017.

Vázquez-de-Aldana, B.R., García-Criado, B., Vicente-Tavera, S. and Zabalgogeazcoa, I., 2013. Fungal endophyte (Epichlö̈ festucae) alters the nutrient content of Festuca rubra regardless of water availability. PloS one. 8(12): p. e84539.

Wang, W.X., Barak, T., Vinocur, B. Shoseyov, O. and Altman, A., 2003. Abiotic resistance and chaperones: possible physiological role of SP1, a stable and stabilizing protein from Populus. In Plant Biotechnology 2002 and Beyond. Springer, Dordrecht. pp. 439-443

Wang, W.X., Vinocur, B., Shoseyov, O. and Altman, A., 2000, July. Biotechnology of plant osmotic stress tolerance physiological and molecular considerations. In IV International Symposium on In Vitro Culture and Horticultural Breeding. 560: pp. 285292.

\section{How to cite this article:}

Prajjal Dey, Diptanu Datta, Debanjana Saha, Selukash Parida and Darshan Panda. 2019. PlantEndophyte Interaction and Its Application to Abiotic Stress Management of Crop Plants. Int.J.Curr.Microbiol.App.Sci. 8(07): 2708-2716. doi: https://doi.org/10.20546/ijcmas.2019.807.332 